\title{
LONGITUDINALLY EXTENSIVE TRANSVERSE MYELITIS AS A PRESENTATION OF SLE FLARE AFTER COVID-19 INFECTION
}

Carlos Eduardo Garcez Teixeira ${ }^{1, \star}$, Daniela Gomes Chicre Oliveira ${ }^{1}$, Marina Ferreira Simões ${ }^{1}$, Isabela Tambelli Pires Cardoso ${ }^{1}$, Yasmin Algazal ${ }^{1}$, Julia Manara Martins ${ }^{1}$, Thiago Bezerra Matias ${ }^{1}$, Alisson Aliel Vigano Pugliesi ${ }^{1}$, Lilian Tereza Lavras Costallat ${ }^{1}$, Simone Appenzeller ${ }^{1}$

1.Universidade Estadual de Campinas, Campinas (SP), Brazil.

*Corresponding author: carlosgarcezt@gmail.com

\section{BACKGROUND}

Systemic lupus erythematosus (SLE) is a chronic autoimmune disease characterized by flare-remission pattern, organ involvement and high morbimortality rate. Neurological involvement may cause serious and permanent damage if not recognized and treated rapidly. Moreover, infection is known as a common trigger of SLE flares and its differentiation with active disease is challenging as they carry similar clinical presentation, thus leading to delayed diagnosis and treatment.

\section{CASE REPORT}

A 31-year-old woman with SLE since 2008 (polyarthritis, oral ulcers, hypocomplementemia and positive antibodies studies: ANA, anti-DNA and Anti-Sm) presented to the emergency department with a 5-day history of new onset paresthesia, paresis in lower extremities bilaterally, urinary and fecal retention and low back pain. She had been in a remission-maintenance treatment with azathioprine (100 mg/day) and hydroxychloroquine ( $5 \mathrm{mg} / \mathrm{kg} /$ day) after lupus nephritis flare for 5 months. Two weeks prior to her admission, she had contacted positive COVID-19 family members and had developed flu-like symptoms. Neurological tests revealed reduced muscle strength and absent deep tendon reflex of the lower extremities. Skin sensibility was altered only for tactile stimulation on both lower limbs. Her mental status, speech, coordination and cranial nerves were normal. During hospitalization, the patient developed headache, fever and neck stiffness, so ceftriaxone ( $4 \mathrm{~g} /$ day) was empirically initiated. However, clinical assessment did not show any improvement on the following days. Cerebrospinal fluid analysis showed an inflammatory pattern. The microbiological screen was negative for bacterial, mycobacterial and fungus both in direct microscopy and cultures. Also, adenosine deaminase was normal and neoplastic cells and oligoclonal bands were negative. Magnetic resonance imaging (MRI) revealed diffuse hyperintense signal on T2-weighted images of the spinal cord affecting its entire extension, from cervical region to conus medullaris without anomalous impregnation by contrast (Figure 1). After a multidisciplinary approach, extensive transverse myelitis associated with SLE activity was considered and treatment with methylprednisolone ( $1 \mathrm{~g} /$ day for 3 days) was initiated. Afterwards, cyclophosphamide $\left(0.5 \mathrm{~g} / \mathrm{m}^{2}\right.$ monthly) and prednisone $(1 \mathrm{mg} / \mathrm{kg} / \mathrm{day})$ were started. The patient had significant clinical recovery, with resolution of the fever and improvement in paresthesia and weakness in the lower limbs in a way she could walk alone again. Then she was discharged to an outpatient follow-up.

\section{CONCLUSION}

Neurological involvement in SLE patients is not rare and the definition between disease activity or an infection event remains a challenge. Early detection and treatment reduces morbimortality.

\section{KEYWORDS}

Systemic lupus erythematosus, COVID-19, Transverse myelitis. 


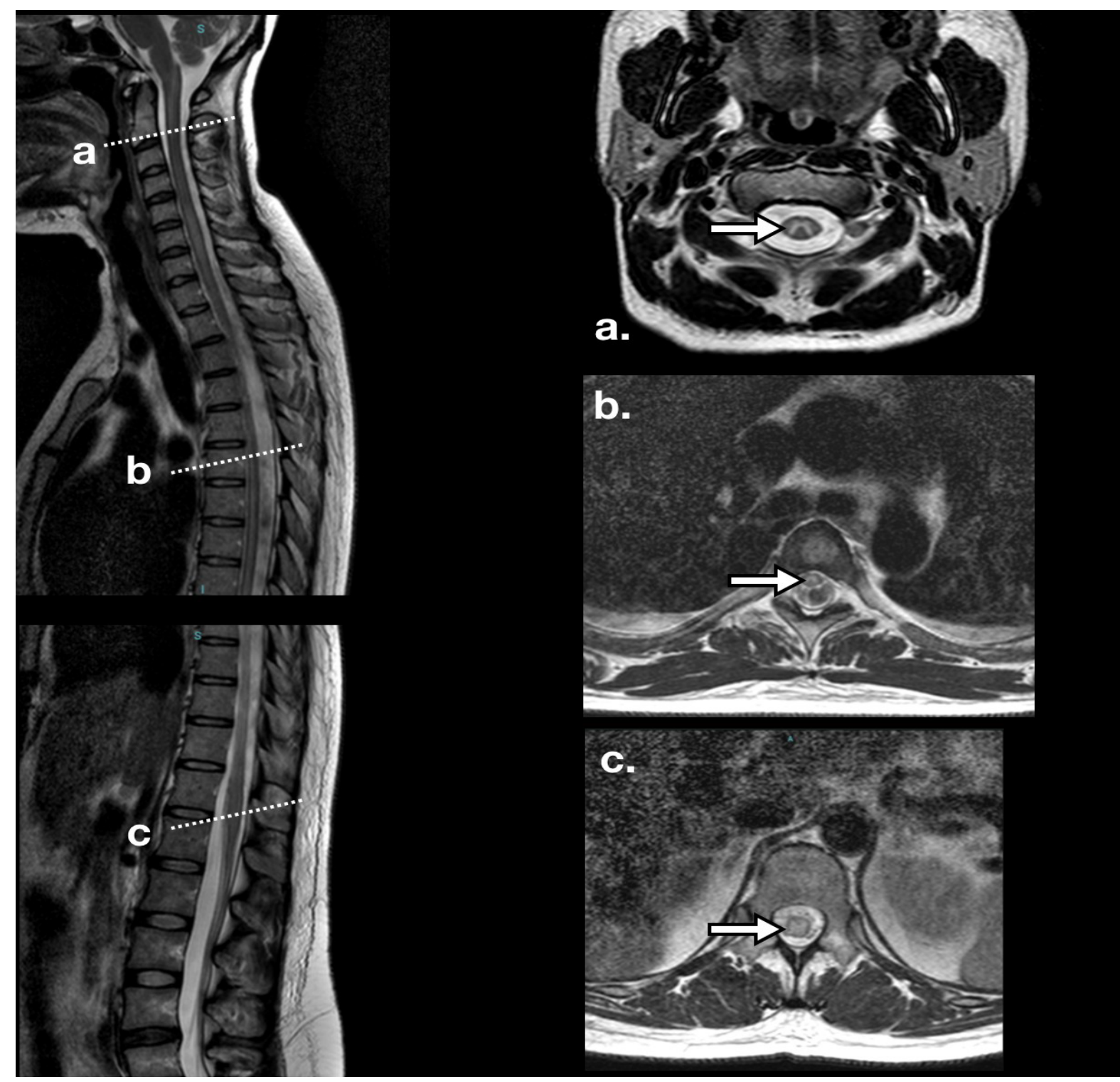

Figure 1. Serial MR images of T2-weighted images. Longitudinally extensive cord lesions seen in cervical (a), thoracic (b) and conus medullaris (c) levels. 